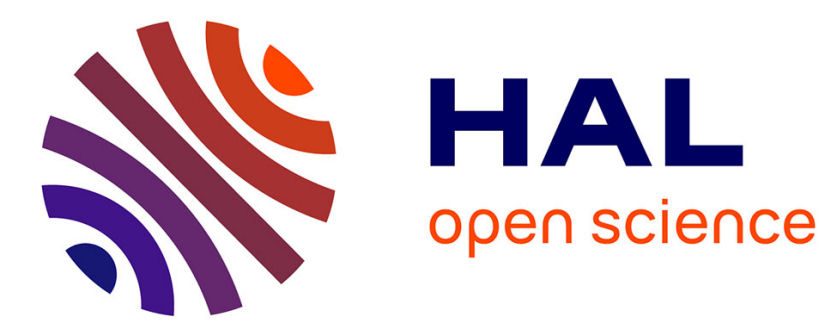

\title{
Mahler measure on Galois extensions
}

Francesco Amoroso

\section{To cite this version:}

Francesco Amoroso. Mahler measure on Galois extensions. Bulletin of the London Mathematical Society, 2016. hal-01575925

\section{HAL Id: hal-01575925 \\ https://hal.science/hal-01575925}

Submitted on 21 Aug 2017

HAL is a multi-disciplinary open access archive for the deposit and dissemination of scientific research documents, whether they are published or not. The documents may come from teaching and research institutions in France or abroad, or from public or private research centers.
L'archive ouverte pluridisciplinaire HAL, est destinée au dépôt et à la diffusion de documents scientifiques de niveau recherche, publiés ou non, émanant des établissements d'enseignement et de recherche français ou étrangers, des laboratoires publics ou privés. 


\title{
MAHLER MEASURE ON GALOIS EXTENSIONS.
}

\author{
FRANCESCO AMOROSO
}

\author{
Laboratoire de mathématiques Nicolas Oresme, CNRS UMR 6139 \\ Université de Caen, Campus II, BP 5186 \\ 14032 Caen Cedex, France
}

\begin{abstract}
We study the Mahler measure of generators of a Galois extension with Galois group the full symmetric group. We prove that two classical constructions of generators gives always algebraic numbers of big height. These results answer a question of C. Smyth and provide some evidence to a conjecture which asserts that the height of such a generator growth to infinity with the degree of the extension.
\end{abstract}

\section{INTRODUCTION}

Let $\alpha$ be a non-zero algebraic number of degree $d$. We let as usual $M(\alpha)$ the Mahler measure of $\alpha$. Thus

$$
\left.M(\alpha)=|\operatorname{lc}(f)| \prod_{j=1}^{d} \max \left(\mid \alpha_{j}\right), 1\right)
$$

where $\operatorname{lc}(f)$ is the leading coefficient of a minimal equation of $\alpha$ over $\mathbb{Z}$ and where $\alpha_{1}, \ldots, \alpha_{d}$ are the conjugates of $\alpha$.

Assume that $\alpha$ is not a root of unity (otherwise $M(\alpha)=1$ ). By an old result of Kronecker $M(\alpha)>1$ and Lehmer [5] asked if we could replace 1 by a real number $>1$ which does not depend on $\alpha$. This problem is still open, the best known result is a theorem of Dobrowolski (see [4]) which proves the lower bound

$$
M(\alpha) \geq 1+c(\varepsilon) d^{-\varepsilon}
$$

for all $\varepsilon>0$, with $c(\varepsilon)>0$ depending only on $\varepsilon$.

Recently, a construction of Smyth gives a renewed interest in lower bounds for the Mahler measure of a generator of a Galois extension (a problem first considered in [1]). In this special case we can considerably improve the above lower bound. Let $\alpha$ be a non-zero algebraic number of degree $d$, not a root unity, such that $\mathbb{Q}(\alpha) / \mathbb{Q}$ is Galois. Then (see $[2]$ )

$$
M(\alpha)^{1 / d} \geq 1+c(\varepsilon) d^{-\varepsilon}
$$

for all $\varepsilon>0$, with $c(\varepsilon)>0$ depending only on $\varepsilon$.

The result of [2] was partially motivated by a problem posed by Smyth during a recent BIRS workshop (see [3, problem 21, p. 17]). Let $n \geq 2$ and $\beta=\beta_{1}, \ldots, \beta_{n}$ be the roots of $z^{n}-z-1$, known to be irreducible for all $n$, and to have Galois group the full symmetric group $\mathfrak{S}_{n}$. Put

$$
\alpha=\beta_{1}^{1} \beta_{2}^{2} \ldots \beta_{n-1}^{n-1} \text {. }
$$

Date: 20 august 2017. 
Then (by an easy consequence of $[6]$ Lemma 1 ) the Galois closure of $\mathbb{Q}(\beta)$ is $\mathbb{Q}(\alpha)$ of degree $d=n$ ! over $\mathbb{Q}$. Smyth computed with Maple the first values of $M(\alpha)^{1 / d}$

\begin{tabular}{|c|r|c|}
\hline$n$ & $d=n !$ & $M(\alpha)^{1 / d}$ \\
\hline 2 & 2 & 1.2720196495 \\
3 & 6 & 1.1509639252 \\
4 & 24 & 1.2428334720 \\
5 & 120 & 1.2292495215 \\
6 & 720 & 1.2846087150 \\
7 & 5040 & 1.2833028970 \\
8 & 40320 & 1.3243452986 \\
9 & 362880 & 1.3307248410 \\
\hline
\end{tabular}

and asked the following questions:

(1) Does anyone know of any smaller values of $M(\alpha)^{1 / d}>1$ for $\alpha$ of degree $d$ with $\mathbb{Q}(\alpha)$ Galois?

(2) Does the above sequence of values $M(\alpha)^{1 / d}$ tend to a limit as $n \rightarrow \infty$ and, if so, what is it?

The quoted result of [2] is related to the first question. One of the aim of this paper is to give an answer to the second question. More generally, in section 3 we show:

Theorem 1.1. Let $\beta$ be an algebraic unit of degree $n$, with algebraic conjugates $\beta_{1}, \ldots, \beta_{n}$. Assume that $\mathbb{Q}\left(\beta_{1}, \ldots, \beta_{n}\right) / \mathbb{Q}$ has Galois group $\mathfrak{S}_{n}$. Let $a_{1}, \ldots, a_{n} \in \mathbb{Z}$ and put

$$
\alpha=\beta_{1}^{a_{1}} \beta_{2}^{a_{2}} \cdots \beta_{n}^{a_{n}} .
$$

Then:

1) $\alpha$ is a generator of $\mathbb{Q}\left(\beta_{1}, \ldots, \beta_{n}\right) / \mathbb{Q}$ if and only if $a_{1}, \ldots, a_{n}$ are pairwise distinct.

2) Put $y_{j}=a_{j}-\frac{1}{n} \sum_{i} a_{i}$. Then

$$
M(\beta)^{c_{n}|\mathbf{y}|_{1}} \leq M(\alpha)^{1 / n !} \leq M(\beta)^{|\mathbf{y}|_{1}}
$$

with $|\mathbf{y}|_{1}=\frac{1}{n} \sum_{j=1}^{n}\left|y_{j}\right|$ and where $c_{n} \sim \sqrt{\frac{2}{\pi n}}$.

3) If $\alpha$ is a generator of $\mathbb{Q}\left(\beta_{1}, \ldots, \beta_{n}\right) / \mathbb{Q}$,

$$
M(\alpha)^{1 / n !} \geq M(\beta)^{(1+o(1)) \sqrt{\frac{n}{8 \pi}}} .
$$

The Mahler measure of a root of $x^{n}-x-1$ is $\geq \theta=1.32 \ldots$, the smallest Pisot's number (the root $>1$ of $x^{3}-x-1$ ). Thus Theorem 1.1 implies that Smyth's sequence tends to $\infty$.

A more classical way to construct a generator for the Galois closure of $\mathbb{Q}(\beta)$ is given by the proof of the Primitive Element Theorem, thus taking a general linear combination of the conjugates of $\beta$. In section 4 we give a proof, based on a simple discriminant argument, of the following partial analogous of Theorem 1.1.

Theorem 1.2. Let $\beta$ be an algebraic integer of degree $n$, with algebraic conjugates $\beta_{1}, \ldots, \beta_{n}$. Assume that $\mathbb{Q}\left(\beta_{1}, \ldots, \beta_{n}\right) / \mathbb{Q}$ has Galois group $\mathfrak{S}_{n}$. Let $a_{1}, \ldots, a_{n} \in \mathbb{Z}$ 
and put

Then:

$$
\alpha=a_{1} \beta_{1}+a_{2} \beta_{2}+\cdots+a_{n} \beta_{n} .
$$

1) $\alpha$ is a generator of $\mathbb{Q}\left(\beta_{1}, \ldots, \beta_{n}\right) / \mathbb{Q}$ if and only if $a_{1}, \ldots, a_{n}$ are pairwise distinct.

2) Let $V(\mathbf{a})$ be the Vandermonde $\prod_{1 \leq i<j \leq n}\left(a_{j}-a_{i}\right)$. Then, if $n \geq 5$,

$$
2^{-\frac{1}{24}}\left(|V(\mathbf{a})| \cdot|\operatorname{disc}(\beta)|^{1 / 2}\right)^{\frac{1}{12 n(n-1)}} \leq M(\alpha)^{1 / n !} \leq|\mathbf{a}|_{1} \cdot M(\beta) .
$$

3) If $\alpha$ is a generator of $\mathbb{Q}\left(\beta_{1}, \ldots, \beta_{n}\right) / \mathbb{Q}$,

$$
M(\alpha)^{1 / n !} \geq(n / 4)^{1 / 48} .
$$

Theorems 1.1 and 1.2 may suggest some speculations on the behavior of $M(\alpha)$ for $\alpha$ a generator of a Galois extension.

Conjecture 1.3. Let $\alpha \in \overline{\mathbb{Q}}$ be a generator of of a Galois extension of degree $d=n$ ! with Galois group the full symmetric group $\mathfrak{S}_{n}$. Then,

$$
M(\alpha)^{1 / d} \geq C(d)
$$

with $C(d) \rightarrow+\infty$ for $d \rightarrow+\infty$.

Equivalently, in terms of the Weil height $h(\alpha)=\frac{1}{d} \log M(\alpha)$,

$$
h(\alpha) \geq c(d)
$$

with $c(d)$ growing to infinity with $d$. This would be the first result of the kind " $h \rightarrow+\infty$ ".

Remark that the assumption on the Galois group is necessary, as a couple of exemples of [2] show: take $\zeta_{e}$ a $e$-root of unity and put $\alpha=1+\zeta_{e}$ or $\alpha=2^{1 / e}+\zeta_{e}$, both of uniformly bounded height.

Aknowledgements. We are indebted to Eric Ricard for a convexity argument which is the key of the proof of Lemma 2.2. We warmly thank Tanguy Rivoal for equality (2.3).

\section{Auxiliary Results}

Let $H_{n}:=\left\{\mathbf{x} \in \mathbb{R}^{n} \mid x_{1}+\cdots+x_{n}=0\right\}$ and, for $\mathbf{x} \in H_{n}$,

$$
|\mathbf{x}|_{1}=\frac{1}{n} \sum_{j=1}^{n}\left|x_{j}\right| .
$$

We remark that $\mathfrak{S}_{n}$ acts on $H_{n}\left(\right.$ by $\left.\sigma(\mathbf{x})=\left(x_{\sigma(1)}, \ldots, x_{\sigma(n)}\right)\right)$.

For $\mathbf{x}, \mathbf{y} \in H_{n}$ we set

$$
s_{n}(\mathbf{x}, \mathbf{y})=\frac{1}{n !} \sum_{\sigma \in \mathfrak{S}_{n}}\left|\frac{1}{n} \sum_{j=1}^{n} x_{\sigma(j)} y_{j}\right| .
$$


Thus $s_{n}$ is symmetric,

$$
s_{n}(\mathbf{x}, \mathbf{y})=s_{n}(\mathbf{y}, \mathbf{x}) .
$$

Moreover, for each $\mathbf{y} \in H_{n}$, the function $\mathbf{x} \mapsto s_{n}(\mathbf{x}, \mathbf{y})$ is a seminorm ${ }^{1}$, stable by the action on $\mathfrak{S}_{n}$.

For $h=1, \ldots, n-1$ we define a vector $\mathbf{z}^{(n, h)} \in H_{n}$ by

$$
z_{j}^{(n, h)}= \begin{cases}\frac{n}{2 h} & \text { if } j=1, \ldots, h \\ -\frac{n}{2(n-h)} & \text { if } j=h+1, \ldots, n\end{cases}
$$

(thus $\left|\mathbf{z}^{(n, h)}\right|_{1}=1$ ). Let also

$$
c_{n}=\min _{0<h, k<n} s_{n}\left(\mathbf{z}^{(n, h)}, \mathbf{z}^{(n, k)}\right) .
$$

The aim of this section is to prove the following proposition.

Proposition 2.1. For $\mathbf{x}, \mathbf{y} \in H_{n} \backslash\{0\}$ we have

$$
c_{n} \leq \frac{s_{n}(\mathbf{x}, \mathbf{y})}{|\mathbf{x}|_{1} \cdot|\mathbf{y}|_{1}} \leq 1
$$

Moreover

$$
c_{n} \sim \sqrt{\frac{2}{\pi n}} .
$$

The above lower bound is clearly sharp, and the upper bound is almost sharp, since

$$
s_{n}\left(\mathbf{z}^{(n, 1)}, \mathbf{z}^{(n, k)}\right)=\frac{n}{2(n-1)}
$$

(see Lemma 2.3 below).

Proposition 2.1 easily implies the lower bound for $M(\alpha)$ in assertion 2) of Theorem 1.1, as we shall see in the next session. In the rest of this section we present the proof of the proposition, which follows from the three lemmas below.

Lemma 2.2. Let $\mathbf{x}, \mathbf{y} \in H_{n} \backslash\{0\}$. Let $h=h(\mathbf{x})$ be the cardinality of the set of $j \in\{1, \ldots, n\}$ such that $x_{j} \geq 0$ and similarly for $k=k(\mathbf{y})$. Then

$$
\frac{s_{n}(\mathbf{x}, \mathbf{y})}{|\mathbf{x}|_{1} \cdot|\mathbf{y}|_{1}} \geq \frac{s_{n}\left(\mathbf{z}^{(n, h)}, \mathbf{y}\right)}{|\mathbf{y}|_{1}} \geq s_{n}\left(\mathbf{z}^{(n, h)}, \mathbf{z}^{(n, k)}\right) \text {. }
$$

Proof. Let us prove the first inequality. Since $\mathbf{x} \mapsto s_{n}(\mathbf{x}, \mathbf{y})$ is homogeneous, we can assume $|\mathbf{x}|_{1}=1$. Let $\mathcal{A}$ be the set of $j \in\{1, \ldots, n\}$ such that $x_{j} \geq 0$. Since $\mathbf{x} \mapsto s_{n}(\mathbf{x}, \mathbf{y})$ is invariant by the action of $\mathfrak{S}_{n}$, we can assume $\mathcal{A}=\{1, \ldots, h\}$. We now use a convexity argument suggested by E. Ricard. Let $G$ be the subgroup of $\sigma \in \mathfrak{S}_{n}$ such that $\sigma(\mathcal{A})=\mathcal{A}$. Then

$$
|G|^{-1} \sum_{\sigma \in G} \sigma(\mathbf{x})=\mathbf{z}^{(n, h)}
$$

and, by the convexity of $\mathbf{x} \mapsto s_{n}(\mathbf{x}, \mathbf{y})$,

$$
s_{n}\left(\mathbf{z}^{(n, h)}, \mathbf{y}\right) \leq s_{n}(\mathbf{x}, \mathbf{y}) .
$$

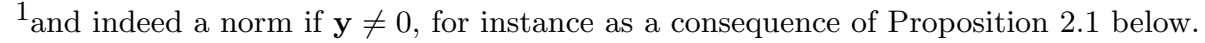


The second inequality follows from the first one and from the symmetry of $s_{n}$ :

$$
\frac{s_{n}\left(\mathbf{z}^{(n, h)}, \mathbf{y}\right)}{|\mathbf{y}|_{1}}=\frac{s_{n}\left(\mathbf{y}, \mathbf{z}^{(n, h)}\right)}{|\mathbf{y}|_{1}} \geq s_{n}\left(\mathbf{z}^{(n, k)}, \mathbf{z}^{(n, h)}\right)=s_{n}\left(\mathbf{z}^{(n, h)}, \mathbf{z}^{(n, k)}\right) .
$$

Lemma 2.3. Let $\mathbf{y} \in H_{n}$ and $h, k \in\{1, \ldots, n\}$. Then

$$
s_{n}\left(\mathbf{z}^{(n, h)}, \mathbf{y}\right)=\frac{n}{2 h(n-h)}\left(\begin{array}{l}
n \\
h
\end{array}\right)^{-1} \sum_{\substack{S \subseteq\{1, \ldots, n\} \\
\operatorname{Card}(S)=h}}\left|\sum_{j \in S} y_{j}\right|
$$

and

$s_{n}\left(\mathbf{z}^{(n, h)}, \mathbf{z}^{(n, k)}\right)=\frac{n^{2}(h-[h k / n])(k-[h k / n])}{2 h k(n-h)(n-k)}\left(\begin{array}{l}n \\ h\end{array}\right)^{-1}\left(\begin{array}{c}k \\ {[h k / n]}\end{array}\right)\left(\begin{array}{c}n-k \\ h-[h k / n]\end{array}\right)$.

Proof. We have

$$
\begin{aligned}
& s_{n}\left(\mathbf{z}^{(n, h)}, \mathbf{y}\right)=\frac{1}{n !} \sum_{\sigma \in \mathfrak{S}_{n}}\left|\frac{1}{n} \sum_{j=1}^{n} z_{j}^{(n, h)} y_{\sigma(j)}\right| \\
& =\frac{1}{n !} \sum_{\sigma \in \mathfrak{S}_{n}}\left|\frac{1}{2 h} \sum_{j=1}^{h} y_{\sigma(j)}-\frac{1}{2(n-h)} \sum_{j=h+1}^{n} y_{\sigma(j)}\right| \\
& =\frac{1}{n !} \sum_{\sigma \in \mathfrak{S}_{n}}\left|\left(\frac{1}{2 h}+\frac{1}{2(n-h)}\right) \sum_{j=1}^{h} y_{\sigma(j)}\right| \\
& =\frac{n}{2 h(n-h)} \frac{1}{n !} \sum_{\sigma \in \mathfrak{S}_{n}}\left|\sum_{j=1}^{h} y_{\sigma(j)}\right| \\
& =\frac{n}{2 h(n-h)}\left(\begin{array}{l}
n \\
h
\end{array}\right)^{-1} \sum_{\substack{S \subseteq\{1, \ldots, n\} \\
\operatorname{Card}(S)=h}}\left|\sum_{j \in S} y_{j}\right| .
\end{aligned}
$$

We now prove the second equality. From the first,

$$
s_{n}\left(\mathbf{z}^{(n, h)}, \mathbf{z}^{(n, k)}\right)=\frac{n}{2 h(n-h)}\left(\begin{array}{l}
n \\
h
\end{array}\right)^{-1} \sum_{\substack{S \subseteq\{1, \ldots, n\} \\
\operatorname{Card}(S)=h}}\left|\sum_{j \in S} z_{j}^{(n, k)}\right| .
$$

For $S \subseteq\{1, \ldots, n\}$ of cardinality $h$ we have

$$
\begin{aligned}
\sum_{j \in S} z_{j}^{(n, k)} & =\frac{n}{2 k}|S \cap\{1, \ldots, k\}|-\frac{n}{2(n-k)}|S \cap\{k+1, \ldots, n\}| \\
& =\left(\frac{n}{2 k}+\frac{n}{2(n-k)}\right)|S \cap\{1, \ldots, k\}|-\frac{n}{2(n-k)}|S| \\
& =\frac{n^{2}}{2 k(n-k)}\left(|S \cap\{1, \ldots, k\}|-\frac{h k}{n}\right) .
\end{aligned}
$$

This gives

$$
s_{n}\left(\mathbf{z}^{(n, h)}, \mathbf{z}^{(n, k)}\right)=\frac{n^{3}}{4 h(n-h) k(n-k)}\left(\begin{array}{l}
n \\
h
\end{array}\right)^{-1} \Sigma_{n, h, k}
$$


with

$$
\begin{aligned}
\Sigma_{n, h, k} & =\sum_{\substack{S \subseteq\{1, \ldots, n\} \\
\operatorname{Card}(S)=h}}|| S \cap\{1, \ldots, k\}\left|-\frac{h k}{n}\right| \\
& =\sum_{j=\max (h+k-n, 0)}^{\min (h, k)}\left(\sum_{\substack{S_{1} \subseteq\{1, \ldots, k\} \\
\operatorname{Card}\left(S_{1}\right)=j}} 1\right)\left(\sum_{\substack{S_{2} \subseteq\{k+1, \ldots, n\} \\
\operatorname{Card}\left(S_{2}\right)=h-j}} 1\right)\left|j-\frac{h k}{n}\right| \\
& =\sum_{j=\max (h+k-n, 0)}^{\min (h, k)}\left(\begin{array}{c}
k \\
j
\end{array}\right)\left(\begin{array}{l}
n-k \\
h-j
\end{array}\right)\left|j-\frac{h k}{n}\right| .
\end{aligned}
$$

We now quote an equality suggested by T. Rivoal. Let $q \in \mathbb{Z}$ with

$$
\max (h+k-n, 0) \leq q \leq \min (h, k) .
$$

Then

$$
\sum_{j=\max (h+k-n, 0)}^{q}\left(\begin{array}{l}
k \\
j
\end{array}\right)\left(\begin{array}{l}
n-k \\
h-j
\end{array}\right)\left(\frac{h k}{n}-j\right)=\frac{1}{n}(h-q)(k-q)\left(\begin{array}{l}
k \\
q
\end{array}\right)\left(\begin{array}{l}
n-k \\
h-q
\end{array}\right) .
$$

This formula can be easily verified by induction on the parameter $q$. It shows that

$$
\sum_{j=\max (h+k-n, 0)}^{\min (h, k)}\left(\begin{array}{l}
k \\
j
\end{array}\right)\left(\begin{array}{l}
n-k \\
h-j
\end{array}\right)\left(\frac{h k}{n}-j\right)=0
$$

and that

$$
\begin{aligned}
\Sigma_{n, h, k} & =2 \sum_{j=\max (h+k-n, 0)}^{[h k / n]}\left(\begin{array}{l}
k \\
j
\end{array}\right)\left(\begin{array}{l}
n-k \\
h-j
\end{array}\right)\left(\frac{h k}{n}-j\right) \\
& =\frac{2}{n}(h-[h k / n])(k-[h k / n])\left(\begin{array}{c}
k \\
{[h k / n]}
\end{array}\right)\left(\begin{array}{c}
n-k \\
h-[h k / n]
\end{array}\right) .
\end{aligned}
$$

Thus

$$
\begin{aligned}
s_{n}\left(\mathbf{z}^{(n, h)}, \mathbf{z}^{(n, k)}\right)= & \frac{n^{3}}{4 h(n-h) k(n-k)}\left(\begin{array}{l}
n \\
h
\end{array}\right)^{-1} \\
& \times \frac{2}{n}(h-[h k / n])(k-[h k / n])\left(\begin{array}{c}
k \\
{[h k / n]}
\end{array}\right)\left(\begin{array}{c}
n-k \\
h-[h k / n]
\end{array}\right) \\
= & \frac{n^{2}(h-[h k / n])(k-[h k / n])}{2 h k(n-h)(n-k)}\left(\begin{array}{l}
n \\
h
\end{array}\right)^{-1}\left(\begin{array}{c}
k \\
{[h k / n]}
\end{array}\right)\left(\begin{array}{c}
n-k \\
h-[h k / n]
\end{array}\right) .
\end{aligned}
$$

In the next lemma we give an asymptotic estimate for

$$
s_{n}\left(\mathbf{z}^{(n, h)}, \mathbf{z}^{(n, k)}\right)=\frac{n^{2}(h-[h k / n])(k-[h k / n])}{2 h k(n-h)(n-k)}\left(\begin{array}{l}
n \\
h
\end{array}\right)^{-1}\left(\begin{array}{c}
k \\
{[h k / n]}
\end{array}\right)\left(\begin{array}{c}
n-k \\
h-[h k / n]
\end{array}\right) .
$$

Lemma 2.4. Let $\left(n_{m}\right)_{n \in \mathbb{N}},\left(h_{m}\right)_{m \in \mathbb{N}},\left(k_{m}\right)_{m \in \mathbb{N}}$ with $0<h_{m}, k_{m}<n_{m}$. Assume

$$
\lim _{m \rightarrow+\infty} n_{m}=+\infty, \quad \lim _{m \rightarrow+\infty} \frac{h_{m}}{n_{m}}=u, \quad \lim _{m \rightarrow+\infty} \frac{k_{m}}{n_{m}}=v
$$


for some $u, v \in[0,1]$. Then

$$
\lim _{m \rightarrow+\infty} 2 \sqrt{2 \pi u v(1-u)(1-v) n_{m}} \cdot s_{n_{m}}\left(\mathbf{z}^{\left(n_{m}, h_{m}\right)}, \mathbf{z}^{\left(n_{m}, k_{m}\right)}\right)=1 .
$$

Proof. By a continuity argument, we can assume $u, v \in(0,1)$. Since

$$
\lim _{m \rightarrow+\infty} \frac{\left[h_{m} k_{m} / n_{m}\right]}{n_{m}}=u v
$$

we have

$$
\frac{n_{m}^{2}\left(h_{m}-\left[h_{m} k_{m} / n_{m}\right]\right)\left(k_{m}-\left[h_{m} k_{m} / n_{m}\right]\right)}{2 h_{m} k_{m}\left(n_{m}-h_{m}\right)\left(n_{m}-k_{m}\right)} \sim \frac{(u-u v)(v-u v)}{2 u v(1-u)(1-v)}=\frac{1}{2} .
$$

For the other factors in $s_{n_{m}}\left(\mathbf{z}^{\left(n_{m}, h_{m}\right)}, \mathbf{z}^{\left(n_{m}, k_{m}\right)}\right)$ we use Stirling's formula, in the following version. Let $\left(n_{m}\right)_{m \in \mathbb{N}},\left(A_{m}\right)_{m \in \mathbb{N}},\left(B_{m}\right)_{m \in \mathbb{N}}$ with $0 \leq B_{m} \leq A_{m}$. Assume $n_{m} \rightarrow+\infty, A_{m} / n_{m} \rightarrow a, B_{m} / n_{m} \rightarrow b$ as $m \rightarrow+\infty$ with $0<b<a$. Then

$$
\left(\begin{array}{l}
A_{m} \\
B_{m}
\end{array}\right) \sim \frac{1}{\sqrt{2 \pi n_{m}}} \times \sqrt{\frac{a}{b(a-b)}} \times\left(\frac{a^{a}}{b^{b}(a-b)^{a-b}}\right)^{n_{m}} .
$$

Thus,

$$
\left(\begin{array}{l}
n_{m} \\
h_{m}
\end{array}\right)^{-1} \sim \sqrt{2 \pi n_{m}} \times \sqrt{u(1-u)} \times\left(u^{u}(1-u)^{1-u}\right)^{n_{m}}
$$

and

$$
\begin{aligned}
& \left(\begin{array}{c}
k_{m} \\
{\left[h_{m} k_{m} / n_{m}\right]}
\end{array}\right) \sim \frac{1}{\sqrt{2 \pi n_{m}}} \times \sqrt{\frac{v}{u v(v-u v)}} \times\left(\frac{v^{v}}{(u v)^{u v}(v-u v)^{v-u v}}\right)^{n_{m}} \\
& =\frac{1}{\sqrt{2 \pi n_{m}}} \times \sqrt{\frac{1}{u v(1-u)}} \times\left(\frac{1}{u^{u v}(1-u)^{v-u v}}\right)^{n_{m}} \\
& \left(\begin{array}{c}
n_{m}-k_{m} \\
h_{m}-\left[h_{m} k_{m} / n_{m}\right]
\end{array}\right) \sim \frac{1}{\sqrt{2 \pi n_{m}}} \times \sqrt{\frac{1-v}{(u-u v)(1-v-u+u v)}} \\
& \times\left(\frac{(1-v)^{1-v}}{(u-u v)^{u-u v}(1-v-u+u v)^{1-v-u+u v}}\right)^{n_{m}} \\
& =\frac{1}{\sqrt{2 \pi n_{m}}} \times \sqrt{\frac{1}{u(1-u)(1-v)}} \\
& \times\left(\frac{1}{u^{u-u v}(1-u)^{1-v-u+u v}}\right)^{n_{m}} .
\end{aligned}
$$


Hence

$$
\begin{aligned}
& s_{n_{m}}\left(\mathbf{z}^{\left(n_{m}, h_{m}\right)}, \mathbf{z}^{\left(n_{m}, k_{m}\right)}\right) \\
& =\frac{n_{m}^{2}\left(h_{m}-\left[h_{m} k_{m} / n_{m}\right]\right)\left(k_{m}-\left[h_{m} k_{m} / n_{m}\right]\right)}{2 h_{m} k_{m}\left(n_{m}-h_{m}\right)\left(n_{m}-k_{m}\right)} \\
& \times\left(\begin{array}{l}
n_{m} \\
h_{m}
\end{array}\right)^{-1}\left(\begin{array}{c}
k_{m} \\
{\left[h_{m} k_{m} / n_{m}\right]}
\end{array}\right)\left(\begin{array}{c}
n_{m}-k_{m} \\
h_{m}-\left[h_{m} k_{m} / n_{m}\right]
\end{array}\right) \\
& \sim \frac{1}{2 \sqrt{2 \pi n_{m}}} \times \sqrt{u(1-u) \times \frac{1}{u v(1-u)} \times \frac{1}{u(1-u)(1-v)}} \\
& \times\left(u^{u}(1-u)^{1-u} \times \frac{1}{u^{u v}(1-u)^{v-u v}} \times \frac{1}{u^{u-u v}(1-u)^{1-v-u+u v}}\right)^{n_{m}} \\
& =\frac{1}{2 \sqrt{2 \pi u v(1-u)(1-v) n_{m}}} .
\end{aligned}
$$

Proof of Proposition 2.1. The upper bound in (2.1) is easily proved:

$$
\begin{aligned}
\frac{1}{n !} \sum_{\sigma \in \mathfrak{S}_{n}}\left|\frac{1}{n} \sum_{j=1}^{n} x_{\sigma(j)} y_{j}\right| & \leq \frac{1}{n !} \sum_{\sigma \in \mathfrak{S}_{n}} \frac{1}{n} \sum_{j=1}^{n}\left|x_{\sigma(j)}\right| \cdot\left|y_{j}\right| \\
& =\frac{1}{n} \sum_{j=1}^{n}\left(\frac{1}{n !} \sum_{\sigma \in \mathfrak{S}_{n}}\left|x_{\sigma(j)}\right|\right)\left|y_{j}\right| \\
& =\frac{1}{n} \sum_{j=1}^{n}|\mathbf{x}|_{1} \cdot y_{j}=|\mathbf{x}|_{1} \cdot|\mathbf{y}|_{1} .
\end{aligned}
$$

The lower bound follows from Lemma 2.2 and from the definition of $c_{n}$. The asymptotic estimate (2.2) for $c_{n}$ is an easy consequence of Lemma 2.4, since

$$
\max _{0 \leq u, v \leq 1} u v(1-u)(1-v)=\frac{1}{16} \text {. }
$$

\section{Proof of Theorem 1.1}

Let us prove the first assertion of the theorem. If $a_{i}=a_{j}$ for some $i \neq j$, then $\alpha$ is not a generator of $\mathbb{Q}\left(\beta_{1}, \ldots, \beta_{n}\right) / \mathbb{Q}$, since $\tau \alpha=\alpha$ for $\tau=(i, j)$. Assume now $a_{1}, \ldots, a_{n}$ pairwise distinct. Let $\sigma$ be a permutation of $\mathfrak{S}_{n}$ such that $\sigma \alpha=\alpha$. Then

$$
\beta_{1}^{a_{1}-a_{\sigma(1)}} \cdots \beta_{n}^{a_{n}-a_{\sigma(n)}}=1,
$$

which implies, by [6, Lemma 1], that $j \mapsto a_{j}-a_{\sigma(j)}$ is constant, say $=\kappa$. For $l=o(\sigma)$ we have $a_{1}=a_{\sigma^{l}(1)}=\cdots=a_{1}+l \kappa$, thus $\kappa=0$ and $\forall j, a_{\sigma(j)}=a_{j}$. Since $a_{1}, \ldots, a_{n}$ are pairwise distinct, $\sigma$ is the identity. 
To prove 2), let $x_{j}=\log \left|\beta_{j}\right| \in \mathbb{R}$ and remark that $\sum_{j} x_{j}=0$ and $\sum_{j}\left|x_{j}\right|=$ $2 \log M(\beta)$. Moreover

$$
\frac{2}{n !} \log M(\alpha)=\frac{1}{n !} \sum_{\sigma \in \mathfrak{S}_{n}}\left|\sum_{j=1}^{n} a_{j} x_{\sigma(j)}\right|=\frac{1}{n !} \sum_{\sigma \in \mathfrak{S}_{n}}\left|\sum_{j=1}^{n} x_{\sigma(j)} y_{j}\right| .
$$

Inequality (2.1) of Proposition 2.1 then gives

$$
c_{n} \frac{2 \log M(\beta)}{n}|\mathbf{y}|_{1} \leq \frac{1}{n} \cdot \frac{2}{n !} \log M(\alpha) \leq \frac{2 \log M(\beta)}{n}|\mathbf{y}|_{1}
$$

with $c_{n} \sim \sqrt{\frac{2}{\pi n}}$. Assertion 2) follows.

To prove the last assertion, we need the following elementary lemma:

Lemma. Let $\mathbf{y} \in H_{n}$ with $y_{j+1}-y_{j} \geq 1$ for $j=1, \ldots, n-1$. Then

$$
|\mathbf{y}|_{1} \geq \frac{n-2}{4} .
$$

Proof. Let $k$ be such that $y_{k} \leq 0<y_{k+1}$. Then, for $j=1, \ldots, k$

$$
y_{j} \leq y_{k}-(k-j) \leq-(k-j)
$$

while

$$
y_{j} \geq y_{k+1}+(j-k-1) \geq j-k-1
$$

for $j=k+1, \ldots, n$. Thus

$$
\begin{aligned}
n|\mathbf{y}|_{1} & =-\sum_{j=1}^{k} y_{j}+\sum_{j=k+1}^{n} y_{j} \geq \sum_{h=0}^{k-1} h+\sum_{h=0}^{n-k-1} h=\frac{(k-1) k}{2}+\frac{(n-k-1)(n-k)}{2} \\
& \geq \frac{n(n-2)}{4} .
\end{aligned}
$$

We can now prove 3$)$. The integers $y_{1}, \ldots, y_{n}$ are pairwise distinct, since $\alpha$ is a generator of $\mathbb{Q}\left(\beta_{1}, \ldots, \beta_{n}\right) / \mathbb{Q}$. Thus we may assume $y_{j+1}-y_{j} \geq 1$ for $j=$ $1, \ldots, n-1$. From the lower bound for $M(\alpha)$ in 2$)$ and from the lemma above we get:

$$
c_{n}|\mathbf{y}|_{1} \sim \sqrt{\frac{2}{\pi n}}|\mathbf{y}|_{1} \geq \sqrt{\frac{2}{\pi n}} \frac{n-2}{4} \sim \sqrt{\frac{n}{8 \pi}} .
$$

\section{Proof of Theorem 1.2}

The proof of the first assertion of Theorem 1.2 is similar to the proof of the corresponding assertion of Theorem 1.1.

For any $\sigma \in \operatorname{Gal}(\mathbb{Q}(\alpha) / \mathbb{Q})$ we have

$$
|\sigma \alpha| \leq|\mathbf{a}|_{1} \max \left\{\left|\beta_{1}\right|, \ldots,\left|\beta_{n}\right|\right\} \leq|\mathbf{a}|_{1} M(\beta)
$$

which shows the upper bound for $M(\alpha)$ in 2$)$. We now prove the lower bound. Let $T_{n}$ be the set of transposition of $\mathfrak{S}_{n}$ and, for $\tau=(i, j) \in T_{n}$, let $A_{\tau}$ be the set of permutation of $\mathfrak{S}_{n}$ with support $\{1, \ldots, n\} \backslash\{i, j\}$ and with no orbits of length 2 . 
Lemma. For $n \geq 3$, let $\Lambda_{n}$ be the set of permutation of $\mathfrak{S}_{n}$ with no orbits of length 1 or 2 . Then $\left|\Lambda_{n}\right| \geq n ! / 12$.

Proof. The inclusion-exclusion principle shows that the set of permutation of $\mathfrak{S}_{n}$ without fixed points has cardinality

$$
\kappa(n):=n !-\frac{n !}{1 !}+\frac{n !}{2 !}-\cdots+(-1)^{n} \frac{n !}{n !} .
$$

Notice that $1 / 3 \leq \kappa(n) / n ! \leq 1 / 2$ for $n \geq 3$. Again by the inclusion-exclusion principle

$$
\left|\Lambda_{n}\right| \geq \kappa(n)-\frac{n(n-1)}{2} \kappa(n-2) \geq \frac{n !}{3}-\frac{n(n-1)}{2} \cdot \frac{(n-2) !}{2}=\frac{n !}{12} .
$$

By the lemma above (recall that $n \geq 5$ ) the sets $A_{\tau}$ have all cardinality $\left|\Lambda_{n-2}\right| \geq$ $\frac{(n-2) !}{12}$. Let

$$
\Delta=\prod_{\tau \in T_{n}} \prod_{\sigma \in A_{\tau}}|\tau \sigma \alpha-\sigma \alpha|
$$

For $\tau=(i, j) \in T_{n}$ with $1 \leq i<j \leq n$ and $\sigma \in A_{\tau}$ we have

$$
\tau \sigma \alpha-\sigma \alpha=\left(a_{j}-a_{i}\right)\left(\beta_{i}-\beta_{j}\right)
$$

since the support of $\sigma$ is disjoint from $\{i, j\}$. Thus

$$
\Delta=\left(|V(\mathbf{a})| \cdot|\operatorname{disc}(\beta)|^{1 / 2}\right)^{\left|\Lambda_{n-2}\right|} .
$$

Let $\tau, \tau^{\prime} \in T_{n}$ and let $\sigma \in A_{\tau}, \sigma^{\prime} \in A_{\tau}$. Then $\tau \sigma \neq \sigma^{\prime}$ (since the supports of $\tau \sigma$ $\sigma^{\prime}$ are not the same). If $\tau=\tau^{\prime}$ and $\sigma \neq \sigma^{\prime}$ then $\tau \sigma \neq \tau^{\prime} \sigma^{\prime}$ and $\sigma \neq \sigma^{\prime}$. Moreover, if $\tau \neq \tau^{\prime}$ then again $\tau \sigma \neq \tau^{\prime} \sigma^{\prime}$ (since $\sigma$ has no orbits of length 2) and $\sigma \neq \sigma^{\prime}$ (since the supports of $\sigma$ and $\sigma^{\prime}$ are not the same). Thus

$$
\Delta \leq \prod_{\tau \in T_{n}} \prod_{\sigma \in A_{\tau}} 2 \max (|\tau \sigma \alpha|, 1) \max (|\sigma \alpha|, 1) \leq 2^{\left|T_{n}\right| \cdot\left|\Lambda_{n-2}\right|} M(\alpha) .
$$

From the two last displayed equations and from the inequality $\left|\Lambda_{n-2}\right| \geq \frac{(n-2) !}{12}$ we get

$$
\begin{aligned}
M(\alpha)^{1 / n !} & \geq\left(2^{-\left|T_{n}\right|}|V(\mathbf{a})| \cdot|\operatorname{disc}(\beta)|^{1 / 2}\right)^{\frac{\left|\Lambda_{n-2}\right|}{n !}} \\
& =2^{-\frac{1}{24}}\left(|V(\mathbf{a})| \cdot|\operatorname{disc}(\beta)|^{1 / 2}\right)^{\frac{1}{12 n(n-1)}} .
\end{aligned}
$$

We finally prove 3 ). Notice that the result is trivial if $n \leq 4$, thus we assume $n \geq 5$. The integers $a_{1}, \ldots, a_{n}$ are pairwise distinct, since $\alpha$ is a generator of $\mathbb{Q}\left(\beta_{1}, \ldots, \beta_{n}\right) / \mathbb{Q}$. Thus we may assume $a_{j+1}-a_{j} \geq 1$ for $j=1, \ldots, n-1$, which implies

$$
|V(\mathbf{a})|=\prod_{i=1}^{n} \prod_{j=i+1}^{n}\left(a_{j}-a_{i}\right) \geq \prod_{i=1}^{n} \prod_{j=i+1}^{n}(j-i)=\prod_{h=1}^{n} h ! .
$$


A computation shows that $\prod_{h=1}^{n} h ! \geq n^{n(n-1) / 4}$. Thus, by 2$)$ and $\operatorname{since}|\operatorname{disc}(\beta)| \geq 1$,

$$
M(\alpha)^{1 / n !} \geq 2^{-\frac{1}{24}}\left(|V(\mathbf{a})| \cdot|\operatorname{disc}(\beta)|^{1 / 2}\right)^{\frac{1}{12 n(n-1)}} \geq(n / 4)^{1 / 48} .
$$

\section{REFERENCES}

1. F. Amoroso and S. David, "Le problème de Lehmer en dimension supérieure", J. Reine Angew. Math. 513 (1999), 145-179.

2. F. Amoroso and D. Masser, "Lower bounds for the height in Galois extensions", Bull. London Math. Soc. 48 (2016), 1008-1012.

3. F. Amoroso, I. Pritsker, C. Smyth and J. Vaaler, "Appendix to Report on BIRS workshop 15w5054 on The Geometry, Algebra and Analysis of Algebraic Numbers: Problems proposed by participants".

Available at http://www.birs.ca/workshops/2015/15w5054/report15w5054.pdf

4. E. Dobrowolski, "On a question of Lehmer and the number of irreducible factors of a polynomial", Acta Arith., 34 (1979), 391-401.

5. D.H. Lehmer, "Factorization of certain cyclotomic functions"; Ann. of Math., 34 (1933), 461-479.

6. C. J. Smyth, "Additive and Multiplicative Relations Connecting Conjugate Algebraic Numbers", J. of Number Theory 23 (1986), 243-254. 